\title{
SOME RARE SPECIES OF MAMMALS FROM BURMA PRESENT IN THE BOMBAY NATURAL HISTORY SOCIETY COLLECTION
}

\author{
Naresh Chaturvedi and Vinod Patil
}

Bombay Natural History Society, Hornbill House, Dr. Sálim Ali Chowk, S.B.S. Road, Mumbai, Maharashtra 400023, India Email: bnhs@bom4.vsnl.net.in

The BNHS had undertaken a systematic survey of the mammals of the Indian subcontinent from 1911 to 1923 . Besides the Indian states the specimens were also collected from various parts of Burma (presently Myanmar) and Ceylon (Sri Lanka). The purpose of the survey was to secure a systematic series of wellpreserved skins and skulls of mammals for a comprehensive study of the status, variation and distribution of the species. The areas from Burma covered under the survey were N. Shan States, Dry Zone, Central Burma, Mt. Popa, S. Tenasserim, Chindwin River, Chin Hills, Pegu, Prome, Mergui Archipelago and Toungoo. The collection of squirrels obtained on both banks of the Chindwin river forms the most magnificent collection, providing striking evidence that the river Chindwin forms a sharp line of demarcation to the eastward and westward extension of the different species of squirrels. All these mammal specimens are kept in dry preservation in the collection of BNHS.

The present paper provides data of rare and endangered mammals collected from Myanmar during the mammal survey of India (1911 to 1923) and a few specimens collected from Burma by BNHS members were also deposited in the Society's collection.

Reg. Date Locality

1. Macaca nemestrina Linnaeus, 1766

Valid name Macaca leonina (Blyth, 1863)

[a] Macaca nemestrina leonina Blyth, 1863

5065 11-xii-1913 Bankachon, Tenasserim

5066 11-xii-1913 Bankachon, Tenasserim

5067 29-xii-1913 Tenasserim

2. Helarctos malayanus Raffles, 1822

5478A05-iii-1916 N. Zameyi River Pegu

3. Felis temmincki Vigors \& Horsfield, 1827 Now placed in Catopuma by Hemmer 1978

[a] Felis temmincki temmincki Vigors \& Horsfield, 1827

$\begin{array}{lllll}6071 & \text { ND } & \text { Laza } & \text { ND } & \text { P.M.R. Leonard } \\ 6072 & \text { ix-1901 } & \text { Pegu } & \text { ND } & \text { C.W. Allen } \\ 6073 & \text { i-1912 } & \text { Akyab } & \text { ND } & \text { A.R. Wellock } \\ 6074 & \text { ND } & \text { Sugan Karen } & \text { ND } & \text { G. Hundley } \\ 6075 & \text { vi-1913 } & \text { Pyinmana } & \text { ND } & \text { C.B. Moggridge }\end{array}$

6075 vi-1913 Pyinmana

[b] Felis temmincki tristis Milne Edwards, 1872

$\begin{array}{lllll}6076 & 1914 & \text { Myitkyina } & \text { ND } & \text { F.C. Lowis } \\ 6080 & 04-\mathrm{ii}-1930 & \text { Haka Felam, Chin Hills } & \text { ND } & \text { T.R. Livesey } \\ 6081 & 19-\mathrm{i}-1919 & \text { Waguul } & \text { ND } & \text { F.V. Clerk }\end{array}$

6081 19-ii-1919 Waguul

Clouded Leopard ND Charles Gray

5. Hystrix hodgsoni Gray, 1847

Now included in Hystrix brachyura

8998 04-x-1913 Allagapph hillays, Sagaing ND Major Owensis

8999 02-ix-1913 Mt. Popa, Upper Burma M G.C. Shortridge

9000 12-xii-1913 Bankachon, V.P.S. Tenasserim M G.C. Shortridge

6. Callosciurus erythraeus Pallas, $1779 \quad$ Pallas's Squirrel

[a] Callosciurus erythraeus atrodorsalis Gray, 1842

7290 13-v-1913 Ataran Riv., Amherst Dist. F J.P. Cook

7291 27-xi-1909 Keng-Tung, S. Shan State ND Cpt.R.D. McGregor

7292 27-xi-1909 Keng-Tung, S. Shan State F Cpt. R.D. McGregor

7293 13-v-1913 Kalaw, Keng-Tung, S. Shan State F Cpt. F.E. Venning

[b] Callosciurus erythraeus erythrogaster Blyth 1842 The Manipuri Squirrel 7315 27-ix-1921 Kanbank F Sálim Ali

7316 10-i-1909 N. Lushai Hills, Chhimlaong M Maj. A. Mears

7317 02-ii-1915 Kabow Valley, 20m.w. of Kindat, F J.M.D. Mackenzie

7318 26-xii-1914 W. Bank Chindwin, 20m.w. of M J.M.D. Mackenzie Kindat

7319 10-v-1914 Nankakank, Kabow valley, Kindat, M J.M.D. Mackenzie

7320 06-v-1914 Chin Hills, 60m.w. of Kindat F J.M.D. Mackenzie

7321 17-viii-1914 Tamanthe, U. Chindwin F S.A. Mcmillan,

F S.A. Mcmillan,

G.C. Shortridge

7323 14-vii-1914 Homalin, U. Chindwin

M S.A. Mcmillan,

G.C. Shortridge

7324 23-iv-1914 Hainggyn, Chin Hills

F J.M.D. Mackenzie

[c] Callosciurus erythraeus intermedius Anderson, 1879

7382 01-v-1923 Putao, Burma ND J.T.O. Bernard

7383 18-iii-1930 Valley of Malillka, Burma M R.W. Burton

[d] Callosciurus erythraeus crotalius Thomas \& Wroughton, 1916 N. Chindwin Squirrel

A detailed note on this squirrel, collected from the Chindwin area was given by Wroughton (1916) in Report 20 of Mammal Survey of India. The collection in Chindwin area was done by S.A. Mcmillan (S.A.M.) and G.C. Shortridge (G.C.S.) and the specimens were identified by Wroughton, R.C.

7388 08-viii-1914 Hkamti, U. Chindwin R. 7389 24-vii-1914 Hkamti, U. Chindwin R. 7390 08-viii-1914 Hkamti, U. Chindwin R. 7391 29-vii-1914 Hkamti, U. Chindwin R. 7392 ND Hkamti, U. Chindwin R. 7393 06-viii-1914 Hkamti, U. Chindwin R. 7394 07-viii-1914 Hkamti, U. Chindwin R. 7395 29-vii-1914 Hkamti, U. Chindwin R. 7396 08-viii-1914 Hkamti, U. Chindwin R. 7397 10-viii-1914 Hkamti, U. Chindwin R. 7398 10-viii-1914 Hkamti, U. Chindwin R. 7399 10-viii-1914 Hkamti, U. Chindwin R. 7400 07-viii-1914 Hkamti, U. Chindwin R. 7401 07-viii-1914 Hkamti, U. Chindwin R. 7402 01-viii-1914 Hkamti, U. Chindwin R. 7403 10-viii-1914 Hkamti, U. Chindwin R. 7404 10-viii-1914 Hkamti, U. Chindwin R. 7405 06-viii-1914 Hkamti, U. Chindwin R. 7406 08-viii-1914 Hkamti, U. Chindwin R. 7407 29-vii-1914 Hkamti, U. Chindwin R.

[e] Callosciurus erythraeus kinneari Thomas \& Wroughton, 1916 Kinnear's Squirrel

7408 29-vi-1914 Tatkon, near Kindat, Chindwin R. 7409 29-vi-1914 Tatkon, near Kindat, Chindwin R. 7410 28-vi-1914 Tatkon, near Kindat, Chindwin R. 7411 04-vii-1914 Tatkon, near Kindat, Chindwin R. 7412 26-vi-1914 Tatkon, near Kindat, Chindwin R. 7413 09-i-1915 Manlaik, S. near Kindat, W. Bank 7414 18-iii-1914 Ahlaw, Kabow Valley, W. Bank

$M \quad$ S.A.M. \& G.C.S. M S.A.M. \& G.C.S. F S.A.M. \& G.C.S. F S.A.M. \& G.C.S. M S.A.M. \& G.C.S. F J.M.D. Mackenzie M J.M.D. Mackenzie

7. Callosciurus flavimanus I. Geoffroy, 1831

Yellow-handed Squirrel Presently based on genetic studies done by Nadler et al. (1975 b.) this species and its subspecies are included in C. erythraeus Corbett \& Hill, 1992

[a] Callosciurus flavimanus phayrei Blyth 1855 7415 24-iii-1927 5M.E. of Toungoo

M J.M.D. Mackenzie 
Reg. Date Locality

7416 10-iii-1927 5M.E. of Toungoo 7417 22-xii-1926 5M.E. of Toungoo 7418 22-xii-1926 5M.E. of Toungoo 7419 09-iii-1927 5M.E. of Toungoo 7420 24-iii-1927 5M.E. of Toungoo 7421 09-ix-1927 6M.E. of Toungoo 7422 22-xi-1927 6M.E. of Toungoo 7423 28-xii-1926 7M.E. of Toungoo 7424 28-xii-1926 7M.E. of Toungoo 7425 28-xii-1926 7M.E. of Toungoo 7426 17-iv-1927 10M.N. of Toungoo 7427 19-iv-1927 10M.N. of Toungoo 7428 07-ii-1927 11M.E. of Toungoo 7429 25-i-1927 15M.N. of Toungoo 7430 29-iii-1927 16M.N. of Toungoo 7431 16-xi-1927 20M.N.E. of Toungoo 7432 19-i-1927 30M.N.E. of Toungoo 7433 19-i-1927 30M.N.E. of Toungoo 7434 19-i-1927 30M.N.E. of Toungoo 7435 13-v-1927 30M.N.E. of Toungoo 7436 13-v-1927 Toungoo

7437 09-viii-1927 Toungoo

7438 13-v-1927 Toungoo

7439 10-iv-1928 Thandaung, Toungoo

7440 15-i-1927 40M.N.E. of Toungoo

7441 16-i-1927 40M.N.E. of Toungoo

7442 14-i-1927 40M.N.E. of Toungoo

7443 24-iv-1913 Gokteik, N. Shan States

7444 17-i-1914 Gokteik, N. Shan States

7445 25-v-1913 Séen, N. Shan States

7446 10-iii-1916 Lashio, N. Shan States

7447 30-vi-1910 Taungyi, S. Shan States

7448 02-viii-1913 East Bank, opp. Kyank myang,

7449 02-viii-1913 East Bank, opp. Kyank myang,

7450 ND Taungyi, S. Shan States

7451 10-ii-1902 Nampandet, Burma

7452 ND Taungyi, S. Shan States

$74531907 \quad$ Taungyi, S. Shan States

74541907 Taungyi, S. Shan States

\section{[b] Callosciurus flavimanus blanfordi Blyth, 1862}

7455 18-v-1913 Gokteik, N. Shan States

7456 27-iv-1913 Gokteik, N. Shan States

7457 24-iv-1913 Gokteik, N. Shan States

7458 24-iv-1913 Gokteik, N. Shan States

7459 23-iv-1913 Gokteik, N. Shan States

7460 25-v-1913 Séen, N. Shan States

7461 30-vii-1913 Hsipaw, N. Shan States

7462 03-viii-1913 E. Bank, Opp. Kyank myang

7463 26-iv-1913 Opp. Kyank myang, N. Shan States

7464 01-viii-1913 Pyaung gaung, N. Shan States

7465 15-v-1913 Opp. Kyank myang, Ngapyiniun

7466 30-vii-1913 N. Shan States

7467 18-v-1913 N. Shan States

7468 24-iv-1913 N. Shan States

7469 26-v-1913 N. Shan States

7470 26-v-1913 Séen, N. Shan States

7471 24-iv-1913 Gokteik, N. Shan States

[c] Callosciurus flavimanus sladeni Anderson, 1871 7472 03-vii-1914 Kindat, U. Chindwin R.

7473 29-vi-1914 Kindat, U. Chindwin R.

7474 30-vi-1914 Kindat, U. Chindwin R.

7475 03-vii-1914 Kindat, U. Chindwin R., E. Bank

7476 29-vi-1914 Kindat, U. Chindwin R., E. Bank

7477 29-vi-1914 Kindat, U. Chindwin R., E. Bank 7478 03-vi-1914 Kindat, U. Chindwin R., E. Bank 7479 29-vi-1914 Kindat, U. Chindwin R., E. Bank 7480 30-vi-1914 Kindat, U. Chindwin R., E. Bank 7481 01-vii-1914 Kindat, U. Chindwin R., E. Bank 7482 29-vi-1914 Kindat, U. Chindwin R., E. Bank 7483 01-vii-1914 Kindat, U. Chindwin R., E. Bank 7484 29-vi-1914 Kindat, U. Chindwin R., E. Bank 7485 29-vi-1914 Kindat, U. Chindwin R., E. Bank 7486 29-vi-1914 Kindat, U. Chindwin R., E. Bank

\section{Sex Collector}

F J.M.D. Mackenzie

F J.M.D. Mackenzie

F J.M.D. Mackenzie

F J.M.D. Mackenzie

F J.M.D. Mackenzie

F J.M.D. Mackenzie

M J.M.D. Mackenzie

M J.M.D. Mackenzie

F J.M.D. Mackenzie

F J.M.D. Mackenzie

M J.M.D. Mackenzie

F J.M.D. Mackenzie

M J.M.D. Mackenzie

M J.M.D. Mackenzie

M J.M.D. Mackenzie

M J.M.D. Mackenzie

F J.M.D. Mackenzie

MJ J.M.D. Mackenzie

F J.M.D. Mackenzie

ND J.M.D. Mackenzie

M J.M.D. Mackenzie

F J.M.D. Mackenzie

F J.M.D. Mackenzie

M J.M.D. Mackenzie

F J.M.D. Mackenzie

F J.M.D. Mackenzie

F J.M.D. Mackenzie

M G.C. Shortridge

F Cpt. F.E. Venning

F G.C. Shortridge

M ND

ND Cpt. S. Lightfoot

M G.C. Shortridge

F G.C. Shortridge

ND ND

ND H.A. Harrington

ND ND

ND H. Lightfool

ND $H$. Lightfool

$\begin{array}{cc}\text { M } & \text { G.C. Shortridge } \\ \text { M } & \text { G.C. Shortridge } \\ \text { M } & \text { G.C. Shortridge } \\ \text { M } & \text { G.C. Shortridge } \\ \text { M } & \text { G.C. Shortridge } \\ \text { M } & \text { G.C. Shortridge } \\ \text { M } & \text { J.P. Cook } \\ \text { M } & \text { G.C. Shortridge } \\ \text { F } & \text { G.C. Shortridge } \\ & \\ \text { M } & \text { G.C. Shortridge } \\ \text { M } & \text { G.C. Shortridge } \\ \text { M } & \text { G.C. Shortridge } \\ \text { M } & \text { G.C. Shortridge } \\ \text { F } & \text { G.C. Shortridge } \\ \text { M } & \text { G.C. Shortridge } \\ \text { F } & \text { G.C. Shortridge } \\ \text { F } & \text { G.C. Shortridge }\end{array}$

M S.A.M. \& G.C.S.

F S.A.M. \& G.C.S.

$M \quad$ S.A.M. \& G.C.S.

$M \quad$ S.A.M. \& G.C.S.

M S.A.M. \& G.C.S.

$M \quad$ S.A.M. \& G.C.S.

F SS.A.M. \& G.C.S

F S.A.M. \& G.C.S

$M \quad$ S.A.M. \& G.C.S

F S.A.M. \& G.C.S

F S.A.M. \& G.C.S

F S.A.M. \& G.C.S

$M \quad$ S.A.M. \& G.C.S.

$M$ S.A.M. \& G.C.S

F S.M. \& G.C.S.

\section{Reg. Date Locality}

7487 14-v-1915 Kindat, U. Chindwin R., E. Bank 7488 01-vii-1914 Kindat, U. Chindwin R., E. Bank 7489 30-vi-1914 Kindat, U. Chindwin R., E. Bank 7490 28-vi-1914 Kindat, U. Chindwin R., E. Bank 7491 29-vi-1914 Kindat, U. Chindwin R., E. Bank 7492 20-vii-1914 Kindat, U. Chindwin R., E. Bank 7493 29-vi-1914 Kindat, U. Chindwin R., E. Bank 7494 28-vi-1914 Kindat, U. Chindwin R., E. Bank 7495 04-xii-1914 S.E. Kindat, Chindwin R., E. Bank 7496 06-xii-1906 Myrtkyina, N. Burma 7497 ND Burma

[d] Callosciurus flavimanus haringtoni Thomas, 1905 7506 29-viii-1914 Moungku, U. Chindwin 7507 18-vii-1914 Moungku, U. Chindwin 7508 29-viii-1914 Moungku, U. Chindwin 7509 17-vii-1914 Moungku, U. Chindwin 7510 15-vii-1914 Homalin, U. Chindwin 7511 17-ix-1914 Homalin, U. Chindwin 7512 13-vii-1914 Homalin, U. Chindwin 7513 13-vii-1914 Homalin, U. Chindwin 7514 14-vii-1914 Homalin, U. Chindwin 7515 17-vi-1914 Homalin, U. Chindwin 7516 17-vi-1914 Homalin, U. Chindwin 7517 16-viii-1914 Homalin, U. Chindwin 7518 16-viii-1914 Homalin, U. Chindwin 7519 17-viii-1914 Homalin, U. Chindwin 7520 17-viii-1914 Homalin, U. Chindwin 7521 ND Homalin, U. Chindwin 7522 18-vii-1914 Homalin, U. Chindwin 7523 18-vii-1914 Homalin, U. Chindwin 7524 13-vii-1914 Homalin, U. Chindwin 7525 16-vii-1914 Homalin, U. Chindwin 7526 17-vii-1914 Homalin, U. Chindwin 7527 16-vii-1914 Homalin, U. Chindwin 7528 17-vii-1914 Homalin, U. Chindwin 7529 27-vii-1914 U. Chindwin R., E. Bank 7530 27-vii-1914 Homalin, U. Chindwin 7531 13-vii-1914 Homalin, U. Chindwin 7532 21-viii-1914 Tamanthe, U. Chindwin 7533 18-viii-1914 Tamanthe, U. Chindwin

[e] Callosciurus flavimanus rubex Thomas, 1914 7534 10-vi-1914 Lower Chindwin, Burma 7535 17-vi-1914 Lower Chindwin, Burma 7536 08-vi-1914 Lower Chindwin, Burma 7537 10-vi-1914 Lower Chindwin, Burma 7538 12-vi-1914 Lower Chindwin, Burma 7539 18-vi-1914 Lower Chindwin, Burma 7540 ND Lower Chindwin, Burma

\section{Sex Collector}

M ND

M S.A.M. \& G.C.S

F S.A.M. \& G.C.S.

F S.A.M. \& G.C.S.

F S.A.M. \& G.C.S.

M S.A.M. \& G.C.S.

F S.A.M. \& G.C.S.

F S.A.M. \& G.C.S.

J.M.D. Mackenzie

E.C. Townsend

ND

M S.A.M. \& G.C.S.

S.A.M. \& G.C.S.

$M \quad$ S.A.M. \& G.C.S.

M S.A.M. \& G.C.S.

F S.A.M \& G.C.S.

$M \quad$ S.A.M. \& G.C.S.

F S.A.M. \& G.C.S.

$M \quad S . M$ \& G.C.S.

$M \quad$ S.A.M. \& G.C.S.

$M \quad$ S.A.M. \& G.C.S.

$F \quad S A M$ \& G.CS.

$M \quad$ S.A.M. \& G.C.S.

M S.A.M. \& G.C.S.

$M \quad$ S.A.M. \& G.C.S.

$M \quad$ S.A.M. \& G.C.S.

$M \quad$ S.A.M. \& G.C.S.

$M \quad$ S.A.M. \& G.C.S.

$M \quad$ S.A.M. \& G.C.S.

$M \quad$ S.A.M. \& G.C.S.

$M \quad$ S.A.M. \& G.C.S.

F S.A.M. \& G.C.S.

$M \quad$ S.A.M. \& G.C.S.

F S.A.M. \& G.C.S

F J.M.D. Mackenzie

M S.A.M. \& G.C.S.

$M \quad$ S.A.M. \& G.C.S.

F S.A.M. \& G.C.S.

F S.A.M. \& G.C.S.

$M \quad$ S.A.M. \& G.C.S.

$M \quad$ S.A.M. \& G.C.S.

$M \quad$ S.A.M. \& G.C.S.

F S.A.M. \& G.C.S.

F S.A.M. \& G.C.S.

F S.A.M. \& G.C.S.

F S.A.M. \& G.C.S.

[f] Callosciurus flavimanus shortridgei Thomas \& Wroughton, 1916

7541 24-vii-1914 Hkamti, U. Chindwin Riv., Burma F S.A.M. \& G.C.S.

7542 03-viii-1914 Hkamti, U. Chindwin Riv., Burma F S.A.M. \& G.C.S.

7543 24-vi-1914 Hkamti, U. Chindwin Riv., Burma M S.A.M. \& G.C.S.

7544 14-viii-1914 Hkamti, U. Chindwin Riv., Burma F S.A.M. \& G.C.S.

7545 15-viii-1914 Hkamti, U. Chindwin Riv., Burma M S.A.M. \& G.C.S.

7546 26-vii-1914 Hkamti, U. Chindwin Riv., Burma F S.A.M. \& G.C.S.

7547 30-vii-1914 Hkamti, U. Chindwin Riv., Burma F S.A.M. \& G.C.S.

7548 30-vii-1914 Hkamti, U. Chindwin Riv., Burma F S.A.M. \& G.C.S.

7549 25-vi-1914 Hkamti, U. Chindwin Riv., Burma M S.A.M. \& G.C.S.

7550 30-vii-1914 Hkamti, U. Chindwin Riv., Burma F S.A.M. \& G.C.S

7551 25-vii-1914 Hkamti, U. Chindwin Riv., Burma F S.A.M. \& G.C.S.

7552 25-vii-1914 Hkamti, U. Chindwin Riv., Burma M S.A.M. \& G.C.S.

7553 30-vii-1914 Hkamti, U. Chindwin Riv., Burma M S.A.M. \& G.C.S

7554 28-vii-1914 Hkamti, U. Chindwin Riv., Burma F S.A.M. \& G.C.S.

7555 29-vii-1914 Hkamti, U. Chindwin Riv., Burma F S.A.M. \& G.C.S.

7556 25-vii-1914 Hkamti, U. Chindwin Riv., Burma F S.A.M. \& G.C.S.

7557 26-vii-1914 Hkamti, U. Chindwin Riv., Burma F S.A.M. \& G.C.S.

7558 29-vii-1914 Hkamti, U. Chindwin Riv., Burma M S.A.M. \& G.C.S.

7559 02-viii-1914 Hkamti, U. Chindwin Riv., Burma F S.A.M. \& G.C.S.

7560 27-vii-1914 Hkamti, U. Chindwin Riv., Burma M S.A.M. \& G.C.S.

7561 25-vii-1914 Hkamti, U. Chindwin Riv, Burma F S.A.M. \& G.C.S.

7562 01-viii-1914 Hkamti, U. Chindwin Riv., Burma F S.A.M. \& G.C.S.

7563 30-vii-1914 Hkamti, U. Chindwin Riv., Burma F S.A.M. \& G.C.S. 
Reg. Date

Locality

Sex Collector

[g] Callosciurus flavimanus millardi Thomas \& Wroughton, 1916 7564 21-vi-1914 Pyaungbyin, Between Kindat 7565 17-vi-1914 Pyaungbyin, Between Kindat 7566 18-vi-1914 Pyaungbyin, Between Kindat

$\begin{array}{ll}\text { F } & \text { ND } \\ \text { M } & \text { B.N.H.S. Team } \\ \text { M } & \text { B.N.H.S. Team }\end{array}$

8. Callosciurus finlaysoni Horsfield, 1824

[a] Callosciurus finlaysoni ferrugineus F. Cuvier, 1827 7567 10-iv-1927 36M.N. of Toungoo(E) 7568 09-iv-1927 36M.N. of Toungoo 7569 02-12-1927 36M.N. of Toungoo(W) 7570 10-iv-1927 36M.N. of Toungoo(E) 7571 02-xii-1927 36M.N. of Toungoo(W) 7572 02-xii-1927 36M.N. of Toungoo 7573 10-iv-1927 36M.N. of Toungoo(E) 7574 26-iv-1927 36M.N. of Toungoo(E) 7575 09-iv-1927 36M.N. of Toungoo(E) 7576 07-xii-1927 30M.N. of Toungoo(E) 7577 23-viii-1927 30M.N. of Toungoo(E) 7578 26-iv-1927 30M.N. of Toungoo(E) 7579 29-iv-1927 30M.N. of Toungoo(E) 7580 23-viii-1927 30M.N. of Toungoo(E) 7581 29-iv-1927 30M.N.W. of Toungoo 7582 23-iv-1927 30M.N. of Toungoo(W) 7583 28-vi-1927 30M.N. of Toungoo(E) 7584 28-vi-1927 30M.N. of Toungoo(E) 7585 09-iv-1927 36M.N. of Toungoo(E) 7586 29-iv-1927 30M.N.W. of Toungoo 7587 05-i-1928 40M.N.W. of Toungoo(E) 7588 20-i-1927 25M.N. of Toungoo(E) 7589 20-iv-1916 Zavngtu, 34M.N. of Pegu 7590 29-ii-1916 Darv'e, 45M.N. of Pegu 7591 28-ii-1916 Darv'e, 45M.N. of Pegu 7592 02-ii-1917 30M.S.E. of Prome 7593 30-i-1917 30M.S.E. of Prome 7594 18-xi-1916 35M.S.E. of Prome 7595 24-x-1916 35M.S.E. of Prome 7596 07-ix-1913 Mt Popa, Myingyan Dist., U. Burma M 7597 08-ix-1913 Mt Popa, Myingyan Dist., U. Burma M 7598 06-ix-1913 Mt Popa, Myingyan Dist., U. Burma F 7599 30-viii-1913 Mt Popa, Myingyan Dist., U. Burma F 7600 03-ix-1913 Mt Popa, Myingyan Dist., U. Burma F 7604 08-ix-1913 Mt Popa, Myingyan Dist., U. Burma M

[b] Callosciurus finlaysoni germaini Milne-Edwards, 1867 7605 07-iii-1913 Siam

ND E.W. Grotter

9. Callosciurus caniceps Gray, 1842

Golden backed Squirrel [a] Callosciurus caniceps davisoni Bonhote, 1901 7606 15-xii-1913 Bankachon, V.P. S. Tenasserim 7607 22-xii-1913 Bankachon, V.P. S. Tenasserim 7608 15-xii-1913 Bankachon, V.P. S. Tenasserim 7609 03-iii-1914 Tenasserim

7610 18-xii-1913 Bankachon, V.P. S. Tenasserim 7611 13-xii-1913 Bankachon, V.P. S. Tenasserim 7612 18-xii-1913 Bankachon, V.P. S. Tenasserim 7613 18-xii-1913 Bankachon, V.P. S. Tenasserim 7614 22-xii-1913 Bankachon, V.P. S. Tenasserim 7615 18-xii-1913 Bankachon, V.P. S. Tenasserim 7616 03-iii-1914 Tenasserim Town

7617 18-xii-1914 Bankachon, V.P. S. Tenasserim 7618 18-xii-1914 Bankachon, V.P. S. Tenasserim 7619 08-xii-1913 Bankachon, V.P. S. Tenasserim 7620 13-xii-1913 Bankachon, V.P. S. Tenasserim 7621 03-iii-1914 Tenasserim Town

7622 08-xii-1913 Bankachon, V.P. S. Tenasserim 7623 05-xii-1913 Bankachon, V.P. S. Tenasserim 7624 11-iii-1914 Tenasserim Town

7625 25-iii-1914 Thager Hills, Tenasserim River 7626 25-xi-1913 Victoria Point, S. Tenasserim 7627 13-xii-1913 Bankachon, V.P. S. Tenasserim 7628 30-xi-1913 Victoria Point, S. Tenasserim 7629 08-xii-1913 Bankachon, V.P. S. Tenasserim 7630 18-iv-1914 Tagvot, Tenasserim River 7631 15-xii-1913 Bankachon, V.P. S. Tenasserim 7632 10-xii-1913 Bankachon, V.P. S. Tenasserim 7633 18-xii-1913 Bankachon, V.P. S. Tenasserim

\section{$\mathrm{F}$}

M G.C. Shortridge

F G.C. Shortridge

M G.C. Shortridge

M G.C. Shortridge

F G.C. Shortridge

M G.C. Shortridge

F G.C. Shortridge

M G.C. Shortridge

M G.C. Shortridge

M G.C. Shortridge

F G.C. Shortridge

M G.C. Shortridge

F G.C. Shortridge

F G.C. Shortridge

M G.C. Shortridge

F G.C. Shortridge

F G.C. Shortridge

M G.C. Shortridge

F G.C. Shortridge

M G.C. Shortridge

F G.C. Shortridge

M G.C. Shortridge

F G.C. Shortridge

F G.C. Shortridge

F G.C. Shortridge

M G.C. Shortridge

M G.C. Shortridge

\section{Reg. Date Locality}

7634 05-xii-1913 Bankachon, V.P. S. Tenasserim 7635 03-iii-1914 Tenasserim Town

7636 09-xii-1913 Bankachon, V.P.S. Tenasserim

7637 25-xi-1913 Victoria Point, S. Tenasserim

7638 04-ix-1921 Mergui, Mergui Dist.

7639 25-iv-1922 Nathay Mile, Mergui Dist.

7640 15-iv-1922 Nathay Mile, Mergui Dist.

7641 17-iv-1922 Nathay Mile, Mergui Dist.

7642 11-iv-1922 Nathay Mile, Mergui Dist.

7643 11-iv-1922 Nathay Mile, Mergui Dist.

7644 11-iv-1922 Nathay Mile, Mergui Dist.

7645 15-iv-1922 Nathay Mile, Mergui Dist.

7646 28-ix-1921 King Island, Mergui

7647 11-ix-1921 King Island, Mergui

7648 ND Kissaraing Island, Madras Dist.

7649 17-iii-1922 Hesting Island, Mergui

7650 14-iii-1922 Hesting Island, Mergui

7652 25-x-1923 Jawoy

7653 11-iv-1908 Received from British Museum

7654 ND Siam

[b] Callosciurus caniceps sullivanus Miller, 1903

7666 02-iii-1922 Lampi or Sullivan Island, Mergui

[c] Callosciurus caniceps domelicus Miller, 1903 7655 06-i-1922 50ft. Kissaraing Island, Mergui 7656 23-i-1922 100ft. Kissaraing Island, Mergui $7657 \quad 27-\mathrm{i}-1922$

$7658 \quad 06-\mathrm{i}-1922$

7659 28-i-1922 Kissaraing Island, Mergui 100ft. Kissaraing Island, Mergui

7660 09-i-1922 Kissaraing Island, Mergui Kissaraing Island, Mergu

$\begin{array}{ll}\text { M } & \text { C. Primrose } \\ \text { M } & \text { C. Primrose } \\ \text { F } & \text { C. Primrose } \\ \text { M } & \text { C. Primrose } \\ \text { M } & \text { C. Primrose } \\ \text { M } & \text { C. Primrose }\end{array}$

[d] Callosciurus caniceps shanicus Ryley, 1914 7667 04-v-1913 Pyaunggaung, N. Shan States 7668 23-i-1914 Hispaw, N. Shan States 7669 29-iv-1913 Pyaunggaung, N. Shan States 7670 10-v-1913 Pyaunggaung, N. Shan States 7671 01-v-1913 Pyaunggaung, N. Shan States 7672 01-v-1913 Pyaunggaung, N. Shan States 7673 01-v-1913 Pyaunggaung, N. Shan States 7674 06-v-1913 Pyaunggaung, N. Shan States 7675 30-iv-1913 Pyaunggaung, N. Shan States 7676 16-iv-1913 Maymyo, Upper Burma 7677 14-iv-1913 Maymyo, Upper Burma 7678 14-iv-1913 Maymyo, Upper Burma 7679 14-iv-1913 Maymyo, Upper Burma 7680 23-i-1914 Hispaw, N. Shan States 7681 08-iv-1914 Lashio, N. Shan States 7682 10-iii-1916 Lashio, N. Shan States 7683 10-iii-1916 Lashio, N. Shan States 7684 08-iv-1916 Lashio, N. Shan States 7685 01-vi-1913 Kalaw 4600'

7686 ND Taungyi, S. Shan States

$\begin{array}{cl}\text { M } & \text { G.C. Shortridge } \\ \text { M } & \text { F.E.W. Venning } \\ \text { M } & \text { G.C. Shortridge } \\ \text { M } & \text { G.C. Shortridge } \\ \text { M } & \text { G.C. Shortridge } \\ \text { M } & \text { G.C. Shortridge } \\ \text { M } & \text { G.C. Shortridge } \\ \text { M } & \text { G.C. Shortridge } \\ \text { F } & \text { G.C. Shortridge } \\ \text { F } & \text { G.C. Shortridge } \\ \text { F } & \text { G.C. Shortridge } \\ \text { F } & \text { G.C. Shortridge } \\ \text { M } & \text { G.C. Shortridge } \\ \text { F } & \text { F.E.W. Venning } \\ \text { F } & \text { F.E.W. Venning } \\ \text { M } & \text { Cpt. W. Massey } \\ \text { M } & \text { Cpt. W. Massey } \\ \text { M } & \text { Cpt. W. Massey } \\ \text { M } & \text { F.E.W. Venning } \\ \text { ND } & \text { S.S. Lightfool }\end{array}$

10. Callosciurus pygerythrus Geoffroy, $1831 \quad$ Irrawaddy Squirrel As per the revision of Moore \& Tate, 1965: 209, all the subspecies are considered as species C. pygerythrus.

[a] Callosciurus pygerythrus pygerythrus Geoffroy, 1831 7696 27-iii-1927 16M.N. of Toungoo (W) 7697 27-iii-1927 16M.N. of Toungoo (W) 7698 18-v-1927 Toungoo (W) 7699 20-v-1927 Toungoo (W)

7700 24-ii-1927 20M.N. of Toungoo (W)

7701 24-ii-1927 20M.N. of Toungoo (W)

7702 23-iii-1927 Toungoo (W)

7703 29-vi-1927 30M.N. of Toungoo (W)

7704 29-vi-1927 30M.N. of Toungoo (W)

7705 08-iv-1927 36M.N. of Toungoo (W)

7706 17-ii-1927 40M.N. of Toungoo (W)

7707 17-ii-1927 40M.N. of Toungoo (W)

7708 18-ii-1927 40M.N. of Toungoo (W)

7709 13-i-1927 40M.N. of Toungoo (W)

7710 18-ii-1927 40M.N. of Toungoo (W)

7711 15-x-1926 30M.N. of Toungoo

\section{Sex Collector}

F G.C. Shortridge

M G.C. Shortridge

M C. Primrose

$F \quad$ C. Primrose

$F \quad$ C. Primrose

F C. Primrose

F C. Primrose

C. Primrose

C. Primrose

C. Primrose

ND ND

M C. Primrose

ND ND

ND ND

ND W.R. Palmer
$F \quad$ G.C. Shortridge

$F \quad$ C. Primrose 
Reg. Date Locality

7714 08-iv-1927 36M.N. of Toungoo

7715 20-v-1927 Toungoo

7716 27-iii-1927 16M.N. of Toungoo

7717 18-ii-1927 40M.N. of Toungoo

7718 13-i-1927 40M.N. of Toungoo

7719 13-i-1927 40M.N. of Toungoo

7720 23-xii-1926 Toungoo (W)

7721 31-iii-1927 16M.N. of Toungoo (W)

7722 31-iii-1927 16M.N. of Toungoo (W)

7723 30-iii-1927 16M.N. of Toungoo (W)

7724 24-ii-1927 20M.N. of Toungoo (W)

7726 29-vi-1927 30M.N. of Toungoo (W)

7727 21-viii-1927 30M.N. of Toungoo (W)

7728 05-i-1927 30M.N. of Toungoo (W)

7729 18-v-1927 30M.N. of Toungoo (W)

7730 28-vi-1927 30M.N. of Toungoo (W)

7731 26-iv-1927 30M.N. of Toungoo (W)

7732 21-viii-1927 30M.N. of Toungoo (W)

7733 07-iv-1927 36M.N. of Toungoo (W)

7734 07-iv-1927 36M.N. of Toungoo (W)

7735 08-iv-1927 36M.N. of Toungoo (W)

7736 13-iv-1927 20M.N. of Toungoo (W)

7737 13-iv-1927 16M.N. of Toungoo (W)

7738 09-iv-1927 36M.N. of Toungoo (W)

7739 21-viii-1927 30M.N. of Toungoo (W)

7740 19-i-1917 35M.S.E. of Prome

7741 18-xi-1916 35M.S.E. of Prome

7742 28-i-1917 35M.S.E. of Prome

7743 28-i-1917 35M.S.E. of Prome

7744 24-x-1916 35M.S.E. of Prome

7745 23-x-1916 35M.S.E. of Prome

7746 18-xi-1916 35M.S.E. of Prome

7747 18-xi-1916 35M.S.E. of Prome

7748 24-i-1917 35M.S.E. of Prome

7749 29-i-1917 35M.S.E of Prome

7750 29-i-1917 35M.S.E. of Prome

7751 21-x-1916 35M.S.E. of Prome

7752 29-iv-1927 30M.N.W. of Toungoo

7753 18-ix-1916 30M.N.W. of Prome

7754 23-ix-1916 30M.N.W. of Prome

7755 16-ix-1916 30M.N.W. of Prome

7756 21-i-1917 40M.S.E. of Prome

7757 16-ix-1916 30M.S.E. of Prome

7758 20-v-1927 Toungoo (W)

7759A22-ix-1916 40M.S.E. of Prome

7759B 19-iv-1916 25M.N. of Pegu

7857 25-xi-1927 Toungoo (W)

7858 25-xi-1927 Toungoo (W)

7859 04-i-1928 20M.N. of Toungoo (W)

7996 02-v-1916 20M.N. of Toungoo (W)
7712 04-i-1927 40M.N. of Toungoo (W)

7713 18-v-1927 Toungoo

7725 13-iv-1927 20M.N. of Toungoo (W)

\section{Sex Collector}

F H.M.W. Mackenzie

M H.M.W. Mackenzie

M H.M.W. Mackenzie

F H.M.W. Mackenzie

M H.M.W. Mackenzie

F H.M.W. Mackenzie

F H.M.W. Mackenzie

F H.M.W. Mackenzie

M H.M.W. Mackenzie

F H.M.W. Mackenzie

M H.M.W. Mackenzie

M H.M.W. Mackenzie

F H.M.W. Mackenzie

M H.M.W. Mackenzie

M J.M.W. Mackenzie

F J.M.W. Mackenzie

F J.M.W. Mackenzie

F J.M.W. Mackenzie

M J.M.W. Mackenzie

M J.M.W. Mackenzie

M J.M.W. Mackenzie

M J.M.W. Mackenzie

M J.M.W. Mackenzie

F J.M.W. Mackenzie

F J.M.W. Mackenzie

M J.M.W. Mackenzie

M J.M.W. Mackenzie

M J.M.W. Mackenzie

M J.M.W. Mackenzie

MJ J.M.W. Mackenzie

M J.M.W. Mackenzie

M J.M.W. Mackenzie

M J.M.W. Mackenzie

F J.M.W. Mackenzie

M J.M.W. Mackenzie

F J.M.W. Mackenzie

M J.M.W. Mackenzie

F J.M.W. Mackenzie

M J.M.W. Mackenzie

F H.M.W. Mackenzie

M H.M.W. Mackenzie

F H.M.W. Mackenzie

M H.M.W Mackenzie

M J.M.W. Mackenzie

F J.M.W. Mackenzie

F J.M.W. Mackenzie

M J.M.W. Mackenzie

M J.M.W. Mackenzie

M J.M.W. Mackenzie

F J.M.W. Mackenzie

M J.M.W. Mackenzie

M J.M.W. Mackenzie
M J.M.W. Mackenzie

\section{Reg. Date Locality}

7882 26-vi-1914 Tatkon, W. Kindat, Chindwin R. 7883 04-vii-1914 Tatkon, W. Kindat, Chindwin R. 7884 26-vi-1914 Tatkon, W. Kindat, Chindwin R. 7885 26-vi-1914 Tatkon, W. Kindat, Chindwin R. 7886 26-vi-1914 Tatkon, W. Kindat, Chindwin R. 7887 01-vii-1914 Tatkon, W. Kindat, Chindwin R. 7888 02-vii-1914 Tatkon, W. Kindat, Chindwin R. 7889 05-vii-1914 Tatkon, W. Kindat, Chindwin R. 7890 14-vii-1914 Homalin, U. Chindwin 7909 19-vii-1914 Homalin, U. Chindwin 7910 21-vii-1914 Tamanthe, U. Chindwin 7911 10-vii-1914 16Miles above Yu River, Chindwin, W. Bank

7912 10-vii-1914 16Miles above Yu River, Chindwin, W. Bank

7915 01-i-1915 6M.S. Kindat, W. Bank, Chindwin R. F 7916 14-ii-1915 6M.S. Kindat, W. Bank, Chindwin R. M 7917 26-vi-1915 Tatkon, W. Bank, Chindwin 7918 26-vi-1914 Tatkon, W. Bank, Chindwin 7919 30-vi-1914 Tatkon, W. Bank, Chindwin 7920 05-vii-1914 Tatkon, W. Kindat, U. Chindwin

7935 09-i-1915 8M.S. Kindat, W. Bank

7936 09-i-1915 8M.S. Kindat, W. Bank

7937 29-xii-1914 13M.W. Kindat, W. Bank

7938 17-iii-1915 5M.W. Kindat

7939 18-ii-1915 6M.W. Kindat

7940 18-ii-1915 6M.W. Kindat

7941 19-ii-1915 6M.W. Kindat

7942 01-i-1915 6M.S. Kindat, W. Bank, Chindwin R.

7943 27-ii-1915 6M.W. Kindat

7944 11-xii-1914 Kindat, W. Bank of Chindwin R.

7945 17-xii-1914 Kindat, W. Bank of Chindwin R.

7946 12-ii-1915 10M.W. Kindat

7947 00-iv-1915 Chin Hills, 50M.W. Kindat

7948 09-iv-1915 60M.N.W. Kindat

7949 12-ii-1915 10M.W. Kindat

7951 08-vi-1914 Kindat, Lower Chindwin

7952 10-vi-1914 Kindat, Lower Chindwin

7953 10-vi-1914 Kindat, Lower Chindwin

7954 18-vi-1914 Kindat, Lower Chindwin

7955 10-vi-1914 Kindat, Lower Chindwin

7956 11-vi-1914 Kindat, Lower Chindwin

7957 09-vi-1914 Kindat, Lower Chindwin

7958 10-vi-1914 Kindat, Lower Chindwin

7959 18-vi-1914 Kindat, Lower Chindwin

7960 18-vi-1914 Kindat, Lower Chindwin

7961 13-vi-1914 Kindat, Lower Chindwin

7962 08-vi-1914 Kindat, Lower Chindwin

7963 10-vi-1914 Kindat, Lower Chindwin

7964 10-vi-1914 Kindat, Lower Chindwin

7965 17-vi-1914 Kindat, Lower Chindwin

7966 19-vi-1914 Kindat, Lower Chindwin

7978 12-x-1914 150M.S. of Kindat, E. Bank

$$
\text { Chindwin R. }
$$

[b] Callosciurus pygerythrus lokroides Hodgson, 1831 7847 25-xi-1927 Toungoo

7797 01-iv-1921 MongPu Hills, Kengtung, W.of Tista M 7798 04-v-1921 MongPu Hills, Kengtung, W.of Tista M 7799 04-v-1921 MongPu Hills, Kengtung, W.of Tista F 7800 05-v-1921 MongPu Hills, Kengtung, W.of Tista M 7801 06-v-1921 MongPu Hills, Kengtung, W.of Tista F 7802 06-v-1921 MongPu Hills, Kengtung, W.of Tista F 7803 28-iv-1921 MongPu Hills, Kengtung, W. of Tista F 7804 29-iv-1921 MongPu Hills, Kengtung. W.of Tista F 7805 01-v-1921 MongPu Hills, Kengtung, W.of Tista M 7806 06-v-1921 MongPu Hills, Kengtung, W.of Tista M 7807 09-v-1921 MongPu Hills, Kengtung, W.of Tista M

J.M.W. Mackenzie

G.L. Shaw

G.L. Shaw

G.L. Shaw

G.L. Shaw

G.L. Shaw

G.L. Shaw

G.L. Shaw

G.L. Shaw

G.L. Shaw

G.L. Shaw

G.L. Shaw

[c] Callosciurus pygerythrus blythi Tytler, 1854 7875 26-vi-1914 Tatkon, W. Kindat, Chindwin R. 7876 30-vi-1914 Tatkon, W. Kindat, Chindwin R. 7877 01-vii-1914 Tatkon, W. Kindat, Chindwin R. 7878 05-vii-1914 Tatkon, W. Kindat, Chindwin R. 7879 05-vii-1914 Tatkon, W. Kindat, Chindwin R. 7880 06-viii-1914 Tatkon, W. Kindat, Chindwin R. 7881 25-vi-1914 Tatkon, W. Kindat, Chindwin R.
F S.A.M. \& G.C.S

$M \quad$ S.A.M. \& G.C.S.

F S.A.M. \& G.C.S

F S.A.M \& G.C.S.

F S.A.M. \& G.C.S.

M S.A.M. \& G.C.S

M S.A.M. \& G.C.S. [d] Callosciurus pygerythrus janetta Thomas, 1914 7760 14-vii-1913 Mingin, W. Sagaing, Upper Burma M 7761 08-vii-1913 Mingin, W. Sagaing, Upper Burma M 7762 13-vii-1913 Mingin, W. Sagaing, Upper Burma F 7763 14-vii-1913 Mingin, W. Sagaing, Upper Burma M 7764 13-vii-1913 Mingin, W. Sagaing, Upper Burma F 7765 13-vii-1913 Mingin, W. Sagaing, Upper Burma M 7766 08-vii-1913 Mingin, W. Sagaing, Upper Burma F 7767 14-vii-1913 Mingin, W. Sagaing, Upper Burma F 7768 27-vii-1913 Kyank-Myaung, Shwebo Dist., W. Burma

7769 27-vii-1913 Kyank-Myaung, Shwebo Dist., W. Burma

7770 27-vii-1913 Kyank-Myaung, Shwebo Dist., W. Burma

7771 27-vii-1913 Kyank-Myaung, Shwebo Dist., W. Burma

7772 28-viii-1913 Mount Popa, Upper Burma

7773 01-ix-1913 Mount Popa, Upper Burma

7774 29-viii-1913 Mount Popa, Upper Burma

7775 21-ix-1913 Mount Popa, Upper Burma

\section{Sex Collector}

S.A.M. \& G.C.S

$M \quad$ S.A.M. \& G.C.S.

$M \quad$ S.A.M. \& G.C.S.

$M \quad$ S.A.M. \& G.C.S.

$M \quad$ S.A.M. \& G.C.S.

M S.A.M. \& G.C.S.

S.A.M. \& G.C.S.

ND S.A.M. \& G.C.S.

F S.A.M. \& G.C.S.

M S.A.M. \& G.C.S.

F S.A.M. \& G.C.S.

$M \quad$ S.A.M. \& G.C.S.

M S.A.M. \& G.C.S.

J.M.W. Mackenzie

J.M.W. Mackenzie

S.A.M. \& G.C.S.

S.A.M. \& G.C.S.

S.A.M. \& G.C.S.

S.A.M. \& G.C.S.

J.M.D. Mackenzie

J.M.D. Mackenzie

J.M.D. Mackenzie

J.M.D. Mackenzie

J.M.D. Mackenzie

J.M.D. Mackenzie

J.M.D. Mackenzie

J.M.D. Mackenzie

J.M.D. Mackenzie

J.M.D. Mackenzie

J.M.D. Mackenzie

J.M.D. Mackenzie

J.M.D. Mackenzie

J.M.D. Mackenzie

J.M.D. Mackenzie

S.A.M. \& G.C.S.

S.A.M. \& G.C.S.

S.A.M. \& G.C.S.

S.A.M. \& G.C.S.

S.A.M. \& G.C.S.

S.A.M. \& G.C.S.

$M$ S.A.M. \& G.C.S.

F S.A.M. \& G.C.S.

$M \quad$ S.A.M. \& G.C.S.

$M$ S.A.M. \& G.C.S.

$M$ S.A.M \& G.C.S.

S.A.M. \& G.C.S.

F S.A.M. \& G.C.S.

M S.A.M. \& G.C.S.

M S.A.M. \& G.C.S.

$M \quad$ S.A.M. \& G.C.S.

M J.M.D. Mackenzie

G.C. Shortridge

G.C. Shortridge

G.C. Shortridge

G.C. Shortridge

G.C. Shortridge

G.C. Shortridge 


\section{Reg. Date Locality}

7776 13-vii-1913 Mingin, W. Sagaing, Upper Burma M 7777 14-vi-1913 Mingin, W. Sagaing, Upper Burma F 7778 14-vi-1913 Mingin, W. Sagaing, Upper Burma M 7779 08-vi-1913 Mingin, W. Sagaing, Upper Burma M 7780 08-vi-1914 Yin, Lower Chindwin

7781 11-vi-1914 Yin, Lower Chindwin

7782 17-vi-1914 Yin, Lower Chindwin

7783 19-vi-1914 Yin, Lower Chindwin

7784 11-vi-1914 Yin, Lower Chindwin

7785 17-vi-1914 Yin, Lower Chindwin

7786 20-viii-1913 Pyawbye, Yamethin Dist., U. Burma F

7787 30-viii-1913 Pyawbye, Yamethin Dist., U. Burma F

7788 22-viii-1913 Pyawbye, Yamethin Dist., U. Burma F

7789 03-vi-1914 Lower Chindwin

7790 17-x-1913 Pagan

7791 01-vii-1913 Mandalay

7792 00-iv-1932 Sheurus Dist.

7793 00-iv-1932 Sheurus Dist.

7794 00-iv-1932 Sheurus Dist.

7795 15-ii-1932 Kyank, Sheurus Dist.

7796 07-v-1932 Kyank, Sheurus Dist.

[e] Callosciurus pygerythrus owensi Thomas \&Wroughton, 1916 7979 11-viii-1914 Hkamti, E. Bank, U. Chindwin R. M S.A.M. \& G.C.S. 7980 12-viii-1914 Hkamti, E. Bank, U. Chindwin R. M S.A.M. \& G.C.S.

11. Callosciurus macclellandi Horsfield, 1839 [a] Callosciurus macclellandi macclellandi 7133 04-xii-1914 30M.S.E. of Kindat, Burma 7134 05-v-1915 Chin Hills, 60M.W. of Kindat 7135 04-v-1914 Chin Hills, 60M.W. of Kindat 7136 03-v-1915 Chin Hills, 60M.W. of Kindat 7137 05-v-1915 Chin Hills, 60M.W. of Kindat 7138 06-v-1915 Chin Hills, 60M.W. of Kindat 7139 06-v-1915 Chin Hills, 60M.W. of Kindat 7140 06-v-1915 Chin Hills, 60M.W. of Kindat 7140A 12-viii-1914 Hkamti, Upper Chindwin 7141 12-viii-1914 Hkamti, Upper Chindwin 7142 28-vii-1914 Hkamti, Upper Chindwin 7143 29-vii-1914 Hkamti, Upper Chindwin 7144 11-viii-1914 Hkamti, Upper Chindwin 7145 28-vii-1914 Hkamti, Upper Chindwin 7146 19-ii-1914 Mogoung, Myitkyina Dist.

[b] Callosciurus macclellandi barbei Blyth, 1847 7175 30-iv-1914 Tenasserim Village

7176 30-iv-1914 Tenasserim Village

7177 30-iv-1914 Tenasserim Village

7178 30-iv-1914 Tenasserim Village

7179 05-iii-1914 Tenasserim Town

7180 05-iii-1914 Tenasserim Town

7181 05-iii-1914 Tenasserim Town

7183 09-iii-1914 Tenasserim Town

7184 09-iii-1914 Tenasserim Town

7185 13-iii-1914 Tenasserim Town

7186 13-iii-1914 Tenasserim Town

7187 14-iii-1914 Tenasserim Town

7188 15-iii-1914 Tenasserim Town

7189 15-iii-1914 Tenasserim Town

7190 16-iii-1914 Tenasserim Town

7191 16-iii-1914 Tenasserim Town

7192 16-iii-1914 Tenasserim Town

7193 17-iii-1914 Tenasserim Town

7194 01-iv-1914 Thaget, Little Tenasserim River 7195 01-iv-1914 Thaget, Little Tenasserim River 7196 21-iv-1914 Banlaw, Great Tenasserim River 7197 23-iv-1914 Banlaw, Great Tenasserim River 7198 24-iv-1914 Banlaw, Great Tenasserim River 7199 25-iv-1914 Banlaw, Great Tenasserim River 7200 25-iv-1914 Banlaw, Great Tenasserim River 7201 25-iv-1914 Banlaw, Great Tenasserim River 7202 26-iv-1914 Banlaw, Great Tenasserim River 7203 26-iv-1914 Banlaw, Great Tenasserim River 7204 26-iv-1914 Banlaw, Great Tenasserim River 7205 06-ix-1927 6M.E. of Toungoo 7206 08-ix-1927 6M.E. of Toungoo

Himalayan Striped Squirrel

J.M.D. Mackenzie J.M.D. Mackenzie M J.M.D. Mackenzie M J.M.D. Mackenzie M J.M.D. Mackenzie M J.M.D. Mackenzie F J.M.D. Mackenzie F J.M.D. Mackenzie M S.A.M. \& G.C.S

$M \quad$ S.A.M. \& G.C.S

F S.A.M. \& G.C.S.

$M \quad$ S.A.M. \& G.C.S

$M \quad$ S.A.M. \& G.C.S

$M \quad$ S.A.M. \& G.C.S

M Cpt. F.E. Venning

M G.C. Shortridge

M G.C. Shortridge

F G.C. Shortridge

F G.C. Shortridge

M G.C. Shortridge

M G.C. Shortridge

F G.C. Shortridge

M G.C. Shortridge

ND G.C. Shortridge

M G.C. Shortridge

M G.C. Shortridge G.C. Shortridge G.C. Shortridge G.C. Shortridge G.C. Shortridge G.C. Shortridge G.C. Shortridge G.C. Shortridge G.C. Shortridge G.C. Shortridge G.C. Shortridge G.C. Shortridge G.C. Shortridge G.C. Shortridge G.C. Shortridge G.C. Shortridge G.C. Shortridge G.C. Shortridge G.C. Shortridge J.M.D. Mackenzie J.M.D. Mackenzie
Reg. Date Locality

7207 08-ix-1927 6M.E. of Toungoo

7208 17-x-1927 8M.W. of Toungoo

7209 09-xi-1927 6M.E. of Toungoo

7210 23-xi-1927 6M.E. of Toungoo

7211 19-i-1928 30M.N.W. of Toungoo

7212 01-ii-1928 35M.N.W. of Toungoo

7213 ND

Kisserain Island

7215 07-i-1922 Kisserain Island

7216 23-ix-1921 King Island

7217 26-iii-1922 Eindayaza

7218 05-v-1913 Pyaunggaung, N. Shan States

7219 06-v-1913 Zammi River, 100M.S. of Moulmein

\section{Sex Collector}

M J.M.D. Mackenzie

F J.M.D. Mackenzie

M J.M.D. Mackenzie

F J.M.D. Mackenzie

M J.M.D. Mackenzie

M J.M.D. Mackenzie

ND J.M.D. Mackenzie

$M \quad$ C. Primrose

M C. Primrose

M C. Primrose

F Sálim Ali

M G.C. Shortridge

J.P. Cook

The specimens collected from Burma (Myanmar) during the Mammal Survey of India were studied by Wroughton R.C. and others and specific reports were published in the journal of the society. The details of the collection locality and observations are published in mammal survey reports and one should refer the following reports for more details.

Report No. 14 (1914) Kathleen V. Ryley, N. Shan states, Burma. Report No. 16 (1915) Wroughton, R.C., Dry zone central province \& Mt. Popa, Burma.

Report No. 17 (1915) Wroughton, R.C., S. Tenasserim, Burma. Report No. 20 (1916) Wroughton, R.C., Chindwin river, Burma. Report No. 25 (1916) Wroughton, R.C., Chin Hills, Burma.

The scientific results from the mammal survey (No.12) on the squirrels collected from Burma was published by Thomas Oldfield and Wroughton, R.C. in 1924.

\section{REFERENCES}

Corbet, G.B. and J.E. Hill (1992). Mammals of the Indo-Malayan Region- A systematic review. Oxford University Press, Oxford, 488pp.

Ellerman, J.R. and T.C.S. Morrisonscott (1951). Checklist of the Palaearctic and Indian Mammals, 210pp.

Hemmer, H. (1978). The evolutionary systematics of living Felidae: present status and current problems. Carnivore 1(1): 71-79.

Hinton, M.A.C. and H.M. Lindsay (1926). Report No. 41 on Assam \& Mishmi Hills, Bombay Natural History Society's Mammal Survey of India, Burma and Ceylon. Journal of the Bombay Natural History Society 31: 383.

Moore, J.C. and G.H.H. Tate (1965). A study of the diurnal squirrels, Sciurinae, of the Indian and Indo-Chinese subregions. Fieldiana, Zoology, 48:1-351.

Ryley, K.V. (1914). Report No. 14 on N. Shan States, Bombay Natural History Society's Mammal Survey of India, Burma and Ceylon. Journal of the Bombay Natural History Society 22: 710.

Fry, T.B. (1928): Report No. 46 on Toungoo Dist., Bombay Natural History Society's Mammal Survey of India, Burma and Ceylon. Journal of the Bombay Natural History Society 32: 545.

Wilson, D.E. and D.M. Reeder (1993). Mammal Species of the World. 1207pp. Wroughton, R.C. (1915). Report No. 16 on Dry zone, C. Burma and Mt. Popa, Bombay Natural History Society's Mammal Survey of India, Burma and Ceylon. Journal of the Bombay Natural History Society 23: 460.

Wroughton, R.C. (1915). Report No. 17 on S. Tenasserim, Bombay Natural History Society's Mammal Survey of India, Burma and Ceylon. Journal of the Bombay Natural History Society 23: 695.

Wroughton, R.C. (1916). Report No. 20 on Chindwin, Bombay Natural History Society's Mammal Survey of India, Burma and Ceylon. Journal of the Bombay Natural History Society 24: 291.

Wroughton, R.C. (1916). Report No. 25 on Chin Hills, Bombay Natural History Society's Mammal Survey of India, Burma and Ceylon. Journal of the Bombay Natural History Society 24: 758 